\title{
A THEORETICAL STUDY TO DESIGN A MICROWAVE RESONATOR FOR SENSING APPLICATIONS
}

\author{
${ }^{*}$ Russul K. Abdulsattar ${ }^{1}$ \\ Zaid A. Abdul hassain ${ }^{1}$ \\ Taha A. Elwi ${ }^{2}$ \\ 1) Electrical Engineering Department, College of Engineering, Mustansiriyah University, Baghdad, Iraq \\ 2) Communication Engineering Department, College of Engineering, Al-Mammon University, Baghdad, Iraq.
}

\begin{abstract}
This paper presents a microwave sensor design for moisture detection contents in petroleum productions based on metamaterials. The proposed sensor occupies a compacted size of $40 \times 40 \times 1.6 \mathrm{~mm}^{3}$ by using low-cost FR4 substrate. The proposed sensor consists of a transmission line and two split-ring resonators (SRRs) mounted on two sides. The transmission line is coupled to the outer ring of the SRRs to provide two resonant frequencies at $1.03 \mathrm{GHz}$ and $3.04 \mathrm{GHz}$. It is observed that most of the electric fields fringing is come out of the air gap on the SRRs. Two pans are mounted on the sensor to contain the sample under test (SUT). The simulation studies are carried out by using the Computer Simulation Technology (CST) Microwave studio. After introducing the pans, frequency shifts, $\triangle \mathrm{f}_{1}=150 \mathrm{MHz}$ and $\triangle \mathrm{f}_{2}=424 \mathrm{MHz}$, are detected in the resonant frequency which is associated with the perturbation phenomenon when the water concentration is varied from $0 \%$ to $100 \%$. The proposed sensor exhibits a reliable detection percentage of various different amounts of the moisture content in crude oil introductions. Generally, a good detection methodology is discovered by applying the proposed sensor for detecting the crude oil quality productions.
\end{abstract}

Keywords: SRR, moisture contents, crude oil.

\section{Introduction}

Recently, the requirement for accurate low water content measurement in oil have been more and more exigent, because it is very important for evaluating crude oil, transmission, and exportation for this reason great interest has been shown from researchers to introduce technologies for measurement moisture content. In [1,2] proposed microwave technology for water detection in petroleum productions, the benefit from using microwave technologies is the capability to represent materials nondestructively, in a contactless manner, besides posing any health hazard to the personnel. Another method to measure water content in crude oil based on x-ray scattering technology was presented in [3]. In [4], a spectral absorption method was presented to measure moisture content of crude oils. Recently, split ring resonators (SRRs) or complementary split ring resonators (CSRRs) played an essential role in sensing technology

$[5-7,13-$ 16]. Electromagnetic sensors based on that take place in the electromagnetic field when the transducer comes in contact with the SUT for sensing $[5,6]$. By using different loads such as: SRRs and CSRRs in order to generate an electric field along the sensor structure to interact with the SUT. For this reason, the proposed sensors are mounted underneath the SUT in the near field to detect the variation through the changes in the resonant frequency and quality factor [7,8]. In this paper, has been proposed a double of SRRs based on microwave resonator to measure the moisture content in crude oil derivatives. The

*Corresponding Author: ru8kh7@gmail.com 
SRRs are designed to produce two resonant frequencies at $1.03 \mathrm{GHz}$ and $3.04 \mathrm{GHz}$. The proposed sensor has successfully detected the water content in the crude oil and is found able to determine quantities of water. The SUT is mounted in the sensor pans to simulate the real fabricated experimental setup. $S_{21}$ spectra are considered in this study to evaluate and classify SUT properties. Finally, the proposed sensor shows another advantage by containing SUT in pans for reuse purposes.

\section{Theoretical Analysis}

In Figure 1, it is shown that the proposed sensor consists of a transmission line (T.L) and a double SRRs on two sides of T.L. Each SRR is composed of a rectangular metal ring with a split on one side. Two splits are symmetrical about the SRR axis and the center part of the T.L is overlapped on one side from the outer ring of the SRR as shown in Figure 1(a). From the back view as shown in Figure 1(b), the back panel of the substrate is covered with a metallic ground plane. From the front view the load (SRRs) is etched in the same panel of T.L, to take the advantage of the quasi-TEM modes propagation [8]. This mode is described by using an oscillating current along the strip and a magnetic field circulating around T.L [6]. The induced current by the magnetic field is found to be parallel to the proposed SRR axis. Therefore, when the electric energy stored in SSRs is equal to the magnetic energy stored in the SRR loop the resonance frequency could occur [9].

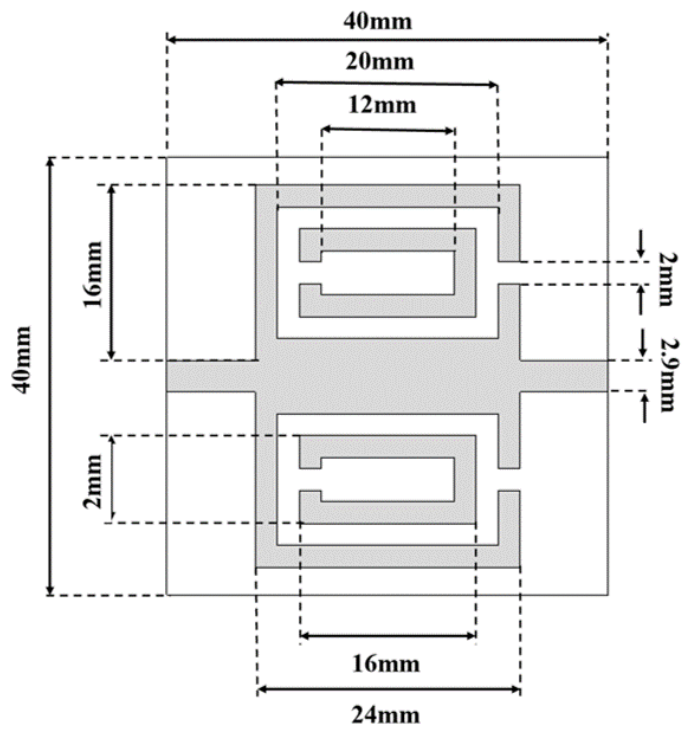

(a)

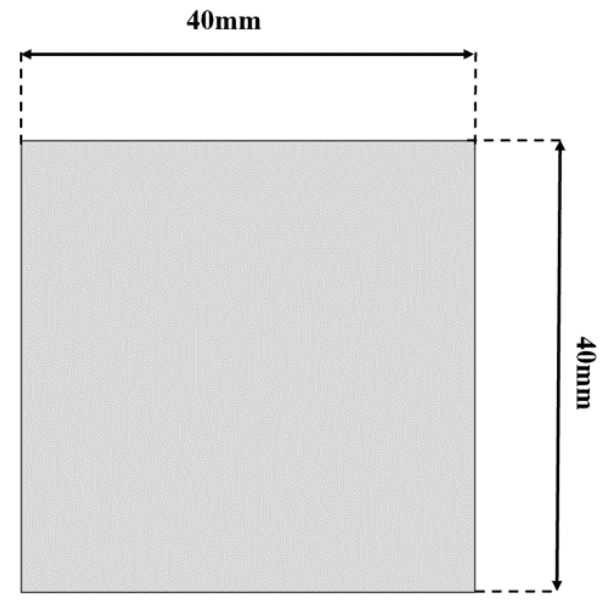

(b)

Figure 1. The proposed sensor geometrical details: (a) front view and (b) back view.

\section{Numerical Analysis}

The proposed sensor is described in section 2 was mounted on FR4 substrate with relative dielectric constant $\varepsilon_{\mathrm{r}}=4.3$ substrate and thickness $\mathrm{t}=1.6 \mathrm{~mm}$. The performance of the sensor is simulated using CST. Figure 2 shown the simulated result of the proposed sensor without pans at two resonant frequencies at $1.03 \mathrm{GHz}$ and $3.04 \mathrm{GHz}$. These two resonance frequencies are appeared because of the direct connection with outer ring [4]. 


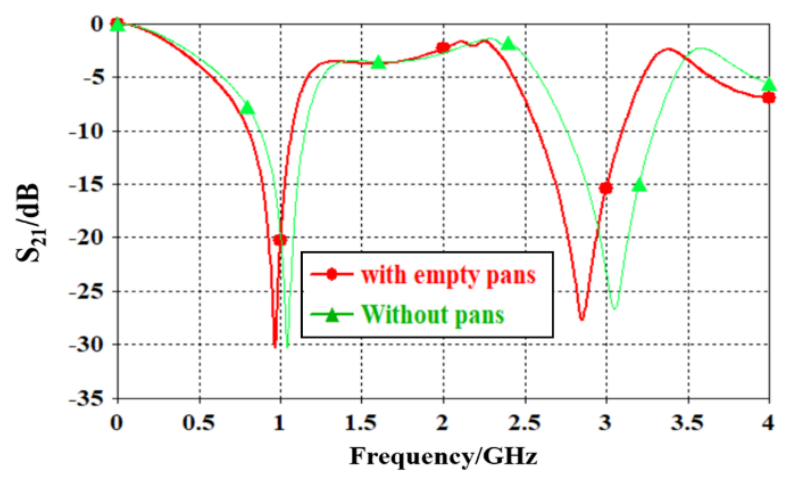

Figure 2. Spectra variations of the proposed sensor with and without pans in term of $S_{21}$.

Now, the electric field distribution is evaluated over the sensor surface to specify the maximum fringing locations. As seen in Figure 3, we found field is mostly distributed on the SRR. At resonance, most of the electric field is distributed on the outer rings of the SRR and across to T.L. Therefore, two pans are placed above the mentioned area on the two sides around T.L. as shown in Figure 4. In such technique, it is ensured that the electric field mostly exposed to the SUT to maintain the detection sensitivity.
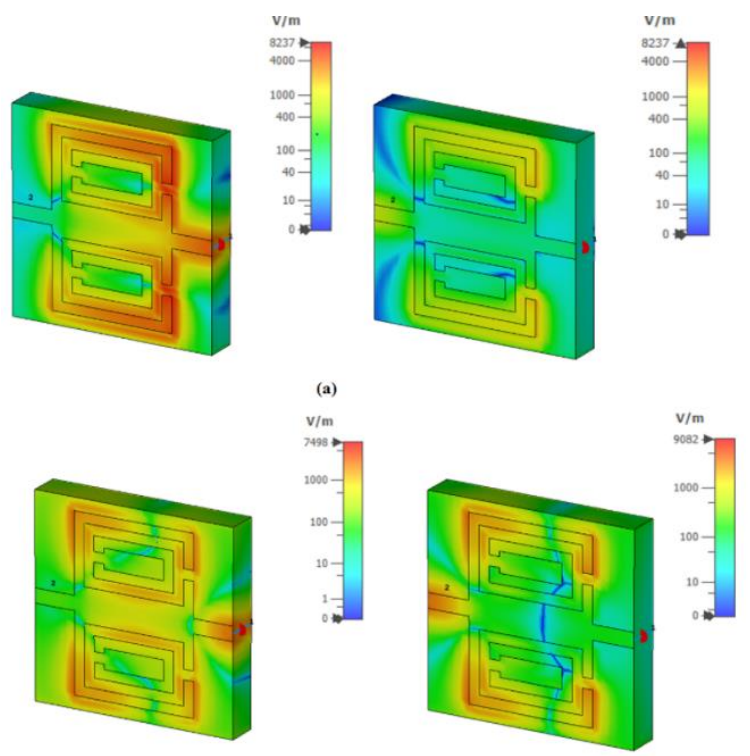

(b)

Figure 3. Simulation electric field from port 1 and port 2 at the resonance frequency: (a) $\mathrm{f}_{1}=1.03 \mathrm{GHz}$ and (b)

$$
\mathrm{f}_{2}=3.04 \mathrm{GHz} \text {. }
$$

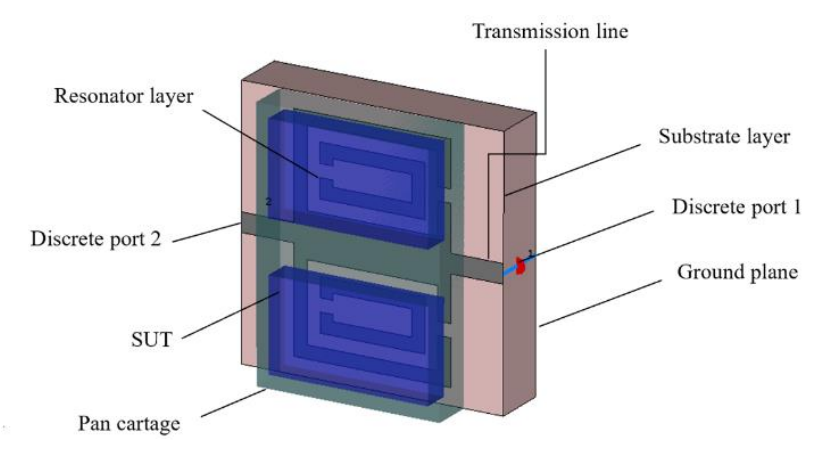

Figure 4. Sensor structure with SUT.

Next, a theoretical study is performed by loading the two pans cartage with different percentages of crude oil to water. However, in this study, the crude oil layer is mounted on the top of the water layer because the crude oil has a less density than water [11]. Both water and crude oil layers have the same area of the pans. After loading the pans with different SUT, the $S_{21}$ spectra are found to be significantly affected as shown in Figure 5. This takes place because of the electric field perturbation in the SUT that leads to a relative $\Delta \mathrm{f}$ according to the SUT dielectric properties [10].

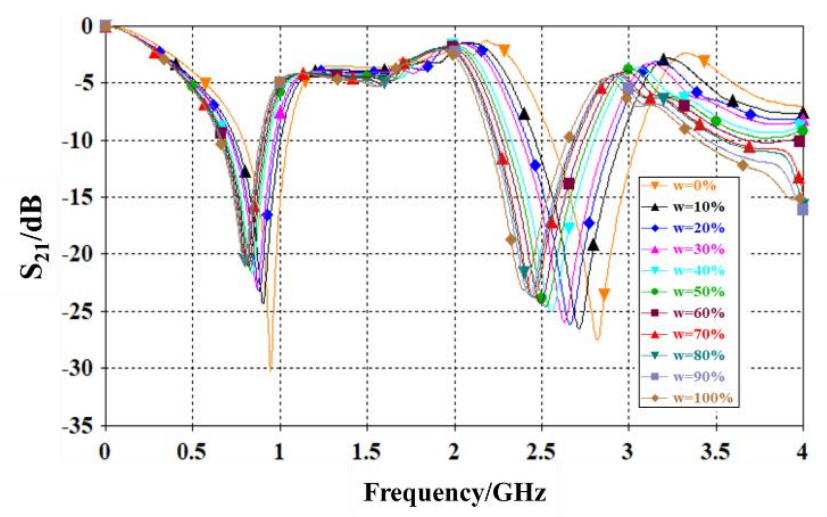

Figure 5. $S_{21}$ spectra variation with different water percentage in the crude oil.

It can be seen in Figure 6, any change in the water content with respect to crude oil percentage is compiled to a relative change in liquid height. Therefore, a parametric study is viewed for three different heights of the pans, $1.2 \mathrm{~mm}, 1.6 \mathrm{~mm}$, and $2 \mathrm{~mm}$, to realize the effects of that on the frequency shift. It is selected at the lowest height, $1.2 \mathrm{~mm}$, with the base equal to $0.4 \mathrm{~mm}$ because the dielectric constant of water is about 80 and 
the dielectric constant of crude oil about 2.44. Therefore, the dielectric constant of a watercontinuous combination is dominated by water content [12].

From the result obtained in table 1, the resonant frequency affected by increasing the water ratio in crude oil, the two resonant frequencies shifted about $\left(\triangle \mathrm{f}_{1}=150 \mathrm{MHz}\right.$ and $\left.\triangle \mathrm{f}_{2}=424 \mathrm{MHz}\right)$ to low frequency, while the bandwidth increasing by increase the water ratio. $S_{21}$ decreases by increasing water from $(-30 \mathrm{~dB}$ to $-20 \mathrm{~dB})$ for the first resonant frequency and from $(-27 \mathrm{~dB}$ to -23 $\mathrm{dB})$ for the second resonant frequency. It has been getting minima and maxima on phase in deg by increasing the water ratio. The quality factor decreased from (42 to 12) for the first region of resonance and from (38 to 20) for the second resonance by increasing the water ratio in crude oil.

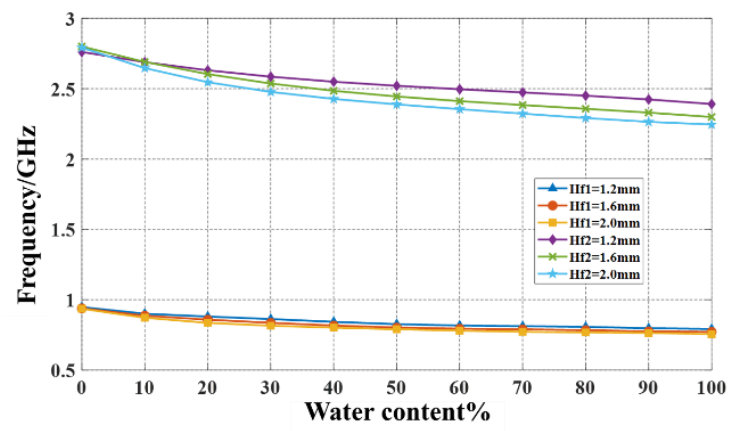

(a)

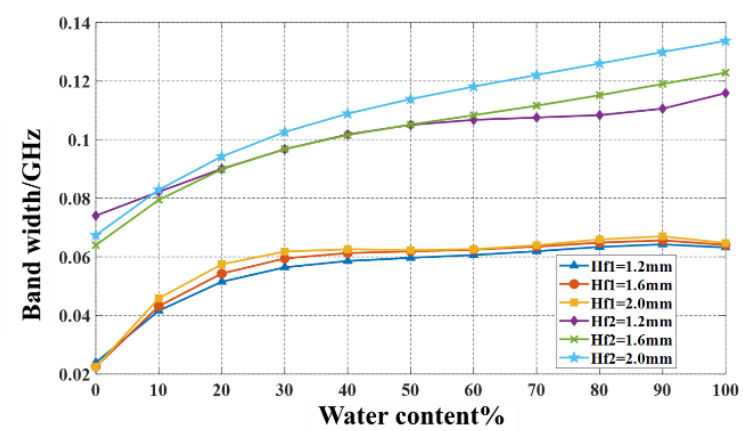

(b)

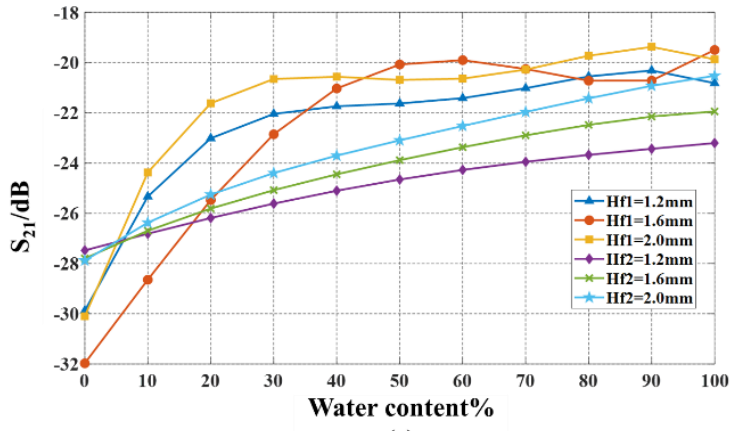

(c)

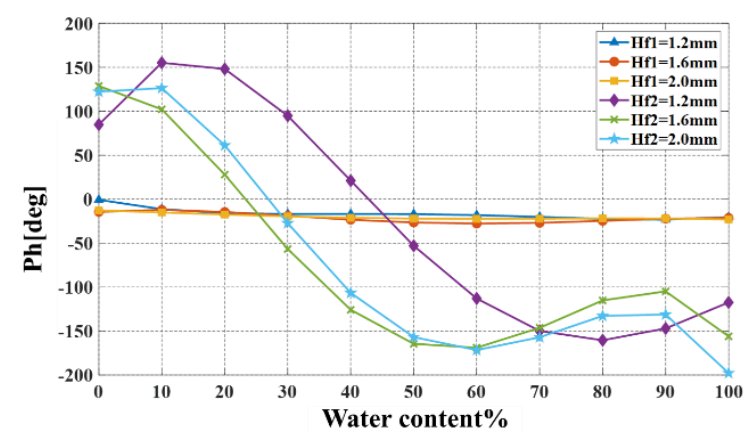

(d)

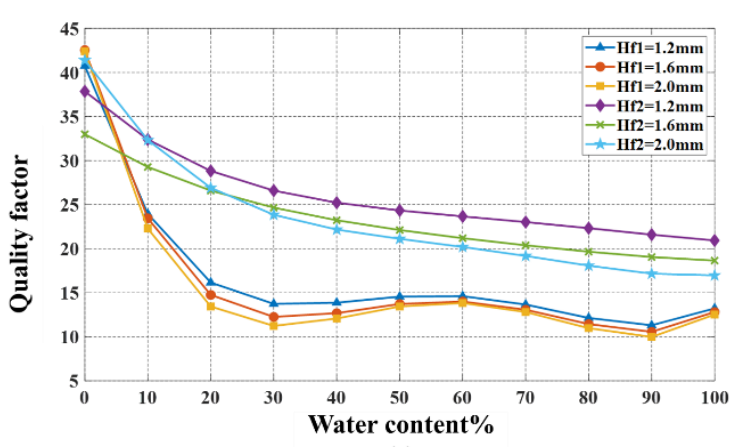

(e)

Figure 6. $S_{21}$ spectra variations with respect to the water percentage variation at different pan heights in terms of:(a) Frequency resonance, (b)bandwidth, (c) $\mathrm{S}_{21}$, (d) phase and (e) quality factor changes.

It can describe the relationship polynomial equation by using MATLAB R2018b between the change in resonant frequency, bandwidth, phase and $S_{21}$ with respect to changes in water construction in the crude oil.

The model that describing the first and second resonant frequencies $\left(f_{1}(x)\right.$ and $\left.f_{2}(x)\right)$ with respect to water content of sample is defined as:

$f_{1}(X)=P_{0}+P_{1} X+P_{2} X^{2}+P_{3} X^{3}$ 
Where $X$ water content in crude oil.

Where $P_{0}, P_{1}, P_{2}$ and $P_{3}$ undefined coefficients that will define a complete relation of dependence of resonance frequency with water content of samples, once they are calculated accurately. From Figure 6(a) it can be solve the equations (1) by curve fitting in MATLAB to find unknown variables.
$P_{1}=-0.003968(-0.005033,-0.002902)$

$P_{2}=0.9441(0.9324,0.9558)$

$P_{3}=0.9441(0.9324,0.9558)$

The same steps have been applied to the second resonant frequency.

$f_{2}(X)=P_{0}+P_{1} X+P_{2} X^{2}+P_{3} X^{3}$

$P_{0}=0.9441(0.9324,0.9558)$

Table 1. Simulated results with different mixtures percentage of water content and crude oil.

\begin{tabular}{ccccccccccccc}
\hline $\mathbf{W \%}$ & $\mathbf{f}_{\mathbf{1}}$ & $\Delta \mathbf{f}_{1}$ & $\mathbf{f}_{2}$ & $\Delta_{\mathbf{f}_{2}}$ & $\mathbf{B . W _ { 1 }}$ & $\mathbf{B . W _ { 2 }}$ & $\left(\mathbf{S}_{21}\right)_{\mathbf{1}}$ & $\left(\mathbf{S}_{21}\right)_{2}$ & $\mathbf{P h}_{1}$ & $\mathbf{P h}_{\mathbf{2}}$ & $\mathbf{Q . F}_{\mathbf{1}}$ & $\mathbf{Q . F}_{\mathbf{2}}$ \\
& $\mathbf{G H z}$ & $\mathbf{G H z}$ & $\mathbf{G H z}$ & $\mathbf{G H z}$ & $\mathbf{G H z}$ & $\mathbf{G H z}$ & $\mathbf{d B}$ & $\mathbf{d B}$ & {$[\mathbf{d e g}]$} & {$[\mathbf{d e g}]$} & & \\
\hline 0 & 0.948 & 0 & 2.82 & 0 & 0.022 & 0.072 & -30.30 & -27.59 & 1.724 & 111.77 & 42.476 & 38.728 \\
10 & 0.9 & 0.048 & 2.563 & 0.257 & 0.045 & 0.084 & -24.29 & -26.56 & -17.6 & 109.1 & 19.688 & 30.177 \\
20 & 0.88 & 0.068 & 2.664 & 0.156 & 0.050 & 0.090 & -23.25 & -26.14 & -13.6 & 114.6 & 17.302 & 29.467 \\
30 & 0.868 & 0.08 & 2.628 & 0.192 & 0.052 & 0.093 & -22.85 & -25.96 & -12.6 & 122.7 & 16.546 & 27.992 \\
40 & 0.836 & 0.112 & 2.556 & 0.264 & 0.059 & 0.1 & -21.71 & -25.02 & -16.8 & 163.04 & 14.018 & 25.519 \\
50 & 0.828 & 0.12 & 2.524 & 0.296 & 0.060 & 0.107 & -21.37 & -24.58 & -19.77 & -170.1 & 13.712 & 23.45 \\
60 & 0.82 & 0.128 & 2.492 & 0.328 & 0.061 & 0.107 & -21.11 & -24.29 & -18.62 & -150.1 & 13.302 & 23.106 \\
70 & 0.812 & 0.136 & 2.456 & 0.364 & 0.062 & 0.107 & -20.94 & -23.81 & -19.69 & -137.8 & 13.027 & 22.874 \\
80 & 0.804 & 0.144 & 2.444 & 0.376 & 0.062 & 0.107 & -20.67 & -23.69 & -22.97 & -135.4 & 12.799 & 22.83 \\
90 & 0.8 & 0.148 & 2.428 & 0.392 & 0.063 & 0.11 & -20.64 & -23.59 & -21.01 & -133.2 & 12.668 & 21.956 \\
100 & 0.792 & 0.156 & 2.396 & 0.424 & 0.063 & 0.116 & -20.63 & -23.12 & -21.44 & -131.2 & 12.396 & 20.626 \\
\hline
\end{tabular}

Where $P_{0}, P_{1}, P_{2}$ and $P_{3}$ equal to $(2.761$ $(2.642,2.881), \quad-0.00807 \quad(-0.01895,0.002811)$, $8.679 \mathrm{e}-05(-0.0001738,0.0003474)$, and $-4.308 \mathrm{e}-$ $07(-2.141 \mathrm{e}-06,1.279 \mathrm{e}-06))$.

The model that describe changes in bandwidth for two resonant frequency with respect to water content in (3) and (4).

$$
\text { B. } W_{1}(X)=P_{0}+P_{1} X+P_{2} X^{2}+P_{3} X^{3}+P_{4} X^{4}
$$

Where $P_{0}, P_{1}, P_{2}, P_{3}$ and $P_{4}$ equal to $(0.02398$ $(0.0179, \quad 0.03006), \quad 0.002263 \quad(0.001326$, $0.003199),-5.57 \mathrm{e}-05$ (-9.672e-05, $-1.468 \mathrm{e}-05)$, 6.186e-07 (-1.258e-08, 1.25e-06)and-2.487e-09 $(-5.617 e-09,6.433 e-10))$
B. $W_{2}(X)=P_{0}+P_{1} X+P_{2} X^{2}+P_{3} X^{3}+P_{4} X^{4}$

Where $P_{0}, P_{1}, P_{2}, P_{3}$ and $P_{4}$ equal to $(0.07296$ $(0.06797, \quad 0.07794), \quad 0.001173 \quad(0.000719$, $0.001627),-1.459 \mathrm{e}-05(-2.546 \mathrm{e}-05,-3.72 \mathrm{e}-06)$ and $7.04 \mathrm{e}-08(-9.279 \mathrm{e}-10,1.417 \mathrm{e}-07))$.

The model that describe changes in $S_{21}$ for two resonant frequency with respect to water content in (5) and (6).

$S_{21}(X)_{1}=P_{0}+P_{1} X+P_{2} X^{2}$ 
Where $P_{0}, P_{1}$ and $P_{2}$ equal to (-28.17 (-30.23, $26.11), 0.2171(0.1212,0.313)$ and -0.001488 ($0.002412,-0.0005642))$.

$S_{21}(X)_{2}=P_{0}+P_{1} X+P_{2} X^{2}$

Where $P_{0}, P_{1}$ and $P_{2}$ equal to $(-27.47$ (-27.79, $27.15), 0.06977 \quad(0.05483,0.08471)$ and $0.0002742(-0.0004181,-0.0001303))$.

The model that describe changes in phase in [deg] for two resonant frequency with respect to water content in (7) and (8).

$P h_{1}(X)=P_{0}+P_{1} X+P_{2} X^{2}+P_{3} X^{3}$

Where $P_{0}, P_{1}, P_{2}$ and $P_{3}$ equal to $(-2.907$ ( -11.66 , $5.849),-0.7244$ (-1.522, 0.0729), 0.01071 ($0.008383,0.02981)$ and $-5.438 \mathrm{e}-05(-0.0001797$, 7.094e-05)).

$P h_{2}(X)=P_{0}+P_{1} X+P_{2} X^{2}+P_{3} X^{3}$

Where $P_{0}, P_{1}, P_{2}$ and $P_{3}$ equal to (101.6 (-63.88, 267.1), $6.262(-8.805,21.33),-0.2779(-0.6388$, $0.083)$ and $0.00195(-0.000418,0.004319))$.

The concentration of water in crude oil has been obtained by compensation the values of resonant frequency, bandwidth, phase, $S_{21}$, and quality factor these values measured by using CST MWS with other values of coefficient where been measured by curve fitting in MATLAB and compensation in equation above from (1 to 8).

Table 2 shows the comparison between the proposed sensor of this work and another microwave resonator reported in the literature.

Table 2. Comparison between microwave resonator

\begin{tabular}{cccc}
\hline Ref. & $\begin{array}{c}\text { Area of } \\
\text { sub/mm }\end{array}$ & $\mathbf{f}_{\mathbf{r}} / \mathbf{G H z}$ & Res. type \\
\hline$[14]$ & $35 \times 35$ & $8-10$ & Oval Wing Res. \\
{$[15]$} & $20 \times 28$ & 2.4 & CSRRs
\end{tabular}

\begin{tabular}{cccc} 
[16] & $25 \times 30$ & 2.4 & $\begin{array}{c}\text { Copm. Circular } \\
\text { Spiral Res. }\end{array}$ \\
This work & $40 \times 40$ & $1.03,3.04$ & SRRs \\
\hline
\end{tabular}

\section{Conclusion}

The proposed sensor has been designed using transmission line technology. It consists of T.L with double SRRs mounted against each other on the two sides. The outer ring of SRR is coupled directly to the middle part of the T.L from one side. The liquid samples are placed inside the pans which are made from the same material of substrate. The proposed sensor is found to operate at two resonant frequencies about 1.09 $\mathrm{GHz}$ and $3.04 \mathrm{GHz}$. The sensor detects various percentages of water contents from (1-100) \% successfully in the crude oil samples. The Results showed the shift in the resonant frequency for the SUTs which mean that the proposed SRR structure has a good sensitivity, high accuracy and can be easily utilized to distinguish any chemical liquids.

\section{Acknowledgements}

This work is supported by the electrical Department/ College of Engineering/ Mustansiriyah University.

\section{Conflict of interest}

The authors declare that the publication of this article causes no conflict of interest.

\section{References}

1. Makeyev, Y.V., Lifanov, A.P., Sovlukov, A.S. (2014). "Microwave measurement of water content in flowing crude oil with improved accuracy". International Crimean Conference Microwave \& Telecommunication Technology, Sevastopol. Ukraine. 7-13 September 2014.

2. Sharma, P., Lao, L., Falcone, G. (2018). "A microwave cavity resonator sensor for waterin-oil measurements”. Sens. Volume 262, 1 June 2018, Pages 200-210. 
3. Luggar, R. D., Key, M. J., Morton, E. J., Gilboy, W. B.(1999). "Energy dispersive xray scatter for measurement of oil/water ratios". Volume 422, Issues 1-3.Pages 938941.

4. Zhao, Y.,Yang, J., Wang, J.Q., Gui, F.X. (2004). "High-accuracy low-water-content measurement of crude oil based on a nearinfrared spectral absorption method". Opt. Eng. Volume 43, Issues 10, Pages 22162217.

5. Galindo-Romera, G., Herraiz-Martínez, F. J., Gil, M., Martínez-Martínez, J. J. SegoviaVargas, D. (2016). "Submersible Printed Split-Ring Resonator-Based Sensor for ThinFilm Detection and Permittivity Characterization". IEEE Sensors Journal. vol. 16, no. 10, pp. 3587-3596.

6. Marques, R., Mesa, F., Martel, J., Medina, F.(2003). "Comparative Analysis of Edgeand Broadside- Coupled Split Ring Resonators for Metamaterial Design - Theory and Experiments". IEEE Transactions on Antennas and Propagation. vol. 51, no. 10, pp. 2572-2581.

7. Lourenço Chuma, E., Iano, Y., Fontgalland, G. Bravo Roger, L. L. (2018). "Microwave Sensor for Liquid Dielectric Characterization Based on Metamaterial Complementary Split Ring Resonator". IEEE. Volume 18, Issue 24, pp 9978-9983.

8. Kang, T., Kim, J., Lee, D., Kang, N. (2016). "Free-space measurement of the complex permittivity of liquid materials at millimeterwave region". Conference on Precision Electromagnetic Measurements (CPEM 2016), Ottawa, ON, Canada. pp. 1-2.

9. Withayachumnankul, W., Jaruwongrungsee, K., Tuantranont, A., Fumeaux, C., Abbott, D. (2013). "Metamaterial-based microfluidic sensor for dielectric characterization". Sens. Actuators A Phys. 189, 233-237.

10. Amer, A. al-Behadili, Iulia, A. M., Norocel C.,Mihaela P. (2020). "Modified Split Ring Resonators Sensor for Accurate Complex Permittivity Measurements of Solid Dielectrics". Sensors. Vol. 20, Iss. 23, 10.3390/s20236855.
11. Qing, L., Bo, Ch., Jinye, P. and Sheng, T. (2019). "A Visual Measurement of Water Content of Crude Oil Based on Image Grayscale Accumulated Value Difference". Sensors. Vol. 19, Iss. 13, 10.3390/s19132963.

12. Zhao, C., Wu, G., Li, Y. (2018). "Measurement of water content of oil-water two-phase flows using dual-frequency microwave method in combination with deep neural network". Measurement. Vol. 131, PP. 92-99.

13. Zaid, A. AbdulHassain, Amer, A. ALBehadili, Adham R. Azeez.(2019). "First order parallel coupled BPF with wideband rejection based on SRR and CSRR". Telkomnika.Vol. 17,Iss. 6,PP. 2704-2712.

14. Abdulkarim, Y.I., Deng, L., Karaaslan, M., Dalgaç Ş., Mahmud, R. H., Ozkan, A. F., Muhammadsharif, F.F., Awl H.N., Huang S., Luo, H.(2020). "The Detection of Chemical Materials with a Metamaterial-Based Sensor Incorporating Oval Wing Resonators". Electronics.Vol.9,Iss.5,10.3390/electronics9 050825.

15. Chuma, E. L., Iano, Y., Fontgalland, G., Roger, L. L. B. (2018). "Microwave sensor for liquid dielectric characterization based on metamaterial complementary split ring resonator", IEEE Sensors J., vol. 18, no. 24, pp. 9978-9983.

16. Zhang, X., Ruan, C., Haq, T. U., Chen, K. (2019). "High-sensitivity microwave sensor for liquid characterization using a complementary circular spiral resonator". Sensors. vol. 19, pp. 787. 